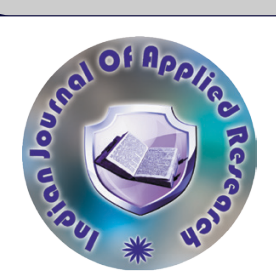

Orthopaedics

\title{
TO EVALUATE THE EFFICACY OF KICK STAND SCREW IN FIXATION OF SUBTROCHANTERIC FEMORAL FRACTURES USING CONTRALATERAL REVERSED DISTAL FEMORAL LOCKING PLATE- PROSPECTIVE SINGLE CENTRIC STUDY
}

\section{Dr Prashant Pandey \\ Dr K K Pandey \\ Dr Ajay Dhanopeeya Dr Surya Prakash Garg*}

SR Orthopaedics Department Of Orthopaedics,Traumatology And Rehabilitation NSCB MCH Jabalpur Mp

Profesor Orthopaedics Department Of Orthopaedics,Traumatology And Rehabilitation NSCB MCH Jabalpur Mp

SR Orthopaedics Department Of Orthopaedics,Traumatology And Rehabilitation NSCB MCH Jabalpur Mp

DNB SR Department Of Orthopaedics,Traumatology And Rehabilitation NSCB MCH Jabalpur Mp *Corresponding Author

ABSTRACT PURPOSE- All type of plates when used for proximal femur fractures always has chances of mechanical failure due to lack of strength of the construct, but addition of 'kick stand screw' has some effects on stability of the construct. Aim of our study is to evaluate the efficacy of kick stand screw in fixation of subtrochanteric fractures using contralateral reversed distal femoral locking plate.

METHOD- 30 patients of subtrochanteric fractures fixed using contralateral reversed distal femoral locking plate. $N\left(1^{\text {st }}\right)=15$ fixed without kick stand screw and other $\mathrm{N}\left(2^{\text {nd }}\right)=15$ fixed using kick stand screw. Calcar screw( Kick Stand Screw) is placed to support calcar region (inferior to the center in AP view and central in Lateral view). In other group screws are placed as oriented in screw holes. Outcome was assessed using Harris Hip Score and VAS score and change in the neck shaft angle is also followed.

RESULT- Union rate was $80 \%$ in 1 st group as compare to $94 \%$ in second group. Mean Harris hip score at the one year follow up was 71.2 in 1 group as compare to 89.2 in $2^{\text {nd }}$ group. Mean Visual analogue scale was 3.2 in first group as compare to 1.6 in second group at final follow up. Mean neck shaft angle at final follow-up was 120 degree in first group as compared to 135 degree in second group. Complication- 1st Group of patients had three failures 2 in the form of screw break down and one in the form of plate bending, all three lend up in to varus deformity but the $2^{\text {nd }}$ group had only one failure in the form of plate bending.

CONCLUSION-In our study cases in which kick stand screw was used they showed better mechanical strength and gave better clinical results. So in proximal femur fractures including subtrochanteric fracture fixation 'kick stand screw' must be used.

\section{KEYWORDS : Kickstand, Calcar, Contralateral, Reversed, DFLP}

\section{INTRODUCTION}

Subtrochanteric fractures are femoral fractures where the fracture occurs below the lesser trochanter up to $5 \mathrm{~cm}$ distally in the shaft of femur ${ }^{1}$. The bone in this region is a thick cortical bone with less vascularity and results in increased potential for healing disturbances. Subtrochanteric fracture is difficult to manage and associated with many complications ${ }^{2}$. The two primary options for treatment of subtrochanteric fractures are intramedullary fixation and extra medullary fixation. However, as several complications may be encountered with all these methods, there is still no ideal treatment choice $^{3,4}$.

Intramedullary nails have been considered the technique of choice for treating simple subtrochanteric femoral fracture ${ }^{5}$. However, various problems are associated with the application of intramedullary nails such as nonunion, delayed union, varus deformity, shaft fracture during surgery, fracture of the trochanter major, perforation in the femoral neck or knee joint and fixation device breakage ${ }^{6}$. Various extra medullary devices have also been developed and used over time for fixing subtrochanteric fractures Such as angled blade plate and the dynamic condylar screw, proximal femur locking plate and sliding ones that have been most widely used in this area ${ }^{7,8,}$

These extra medullary devices when used through a minimally invasive technique, put in the sub muscular zone, preserve both the periosteal and endosteal blood supply in addition to providing stability and environment for fracture healing ${ }^{10}$.

Reversed (upside down) contralateral (plate of opposite limb) distal femoral locking compression plate (DFLCP) as a biomechanically sound alternative extra medullary device for fixation of subtrochanteric fractures in adult, as it provide an added number of screw options for proximal femoral fragments, thus resulting in a more stable construct with higher pull out resistance $\mathrm{e}^{11,12,13}$.

With all locking plates used in the fixation of subtrochanteric femoral fractures varus malreduction is very common complication. To prevent this support of the medial column is advocated. Mechanical support of the inferomedial region of the proximal humerus prevents subsequent loss of reduction. Medial column support can be achieved by anatomic or slightly impacted stable reduction, and placement of a superiorly directed oblique locking screw into the inferomedial region of the proximal femoral fragment. This screw is referred as the Inferomedial Or Calcar Or Kick Stand Screw.

All type of plates when used for proximal femur fractures always has chances of mechanical failure due to lack of strength of the construct, but addition of 'kick stand screw' has some effects on stability of the construct. Aim of our study is to evaluate the efficacy of kick stand screw in fixation of subtrochanteric fractures using contralateral reversed distal femoral locking plate.

\section{MATERIAL AND METHODS}

30 patients of subtrochanteric fractures fixed using contralateral reversed distal femoral locking plate. $\mathrm{N}=15$ fixed without kick stand screw and other $\mathrm{N}=15$ fixed using kick stand screw.

Inclusion Criteria: Age of more than 18 yrs, Subtrochanteric femoral fracture with extension into piriform fossa, Patient with narrow medullary canal, Reverse oblique intertrochanteric fracture

Exclusion Criteria: Pathological fractures, Fracture in children, Old neglected fracture and soft tissue infection at fracture site, Open injury, Previous surgery for proximal femoral fracture, Associated Pelvic fracture, ipsilateral distal femur fracture, On-going CT or RT for any malignancy, periprosthetic fractures.

Surgical approach for the both of the groups was same. In this study we have used lateral approach for proximal femur which was used for plate insertion in sub muscular plane and we also used another incision on lateral aspect of thigh over distal end of plate.

Calcar screw is placed to support calcar region (inferior to the center in AP view and central in Lateral view), which was put in the direction of the neck i.e. in the direction of neck shaft angle. This calcar is screw placed in second or third $6.5 \mathrm{~mm}$ locking cancellous screw hole. This 
calcar screw in known as 'Kick Stand Screw'. In other group of patients all the screws of expanded part of the plate was put as their direction made in the plate i.e. not along the axis of neck of the femur.

Proximal skin incision was made at the centre of proximal femur begin at the tip of the greater trochanter and extending distally about 5 to 7 $\mathrm{cm}$. Subcutaneous tissue cut along the skin incision. Than fascia lata was cut along the skin incision. Now fascia of vastus lateralis splited and we elevate the proximal part of vastus lateralis muscle off the intermuscular septum. Now we release the origin of vastus lateralis muscle from the trochanteric ridge. Now we made sub muscular plane to insert the plate.

Same surgical approach is used for both of the group only difference is that during fixation of proximal fragment we used kick stand screw in second group. Contralateral (left sided plate for right side) Reversed (upside down) plate of appropriate length(mostly 10 hole) was placed with its proximal part at Trochanteric ridge with proximal most screw at the level of superior border of neck. We had first fixed the plate to the proximal fragment with the help of screw placed in second or third $6.5 \mathrm{~mm}$ locking cancellous screw hole. Than we reduce the fracture and fix non locking cortical screw in distal fragment just below fracture to stabilize the fracture and to reduce the bone plate interface. We introduce 4-5 proximal locking screws. While inserting proximal screw, $\mathrm{C}$ arm is used to confirm their placement in the neck in anteroposterior and lateral view. We put 4-5 screws distal to the fracture. Being a locking plate, it did not require any periosteal stripping. For the purpose of fixation in the proximal femur, the target was to drive long screw in two rows of locking screw through the proximal expanded part of locking plate and 4-5 cortical purchased in distal fracture fragment. All wound were closed over drain which was removed after $24 \mathrm{hr}$ of surgery.

\section{Kick Stand Screw}

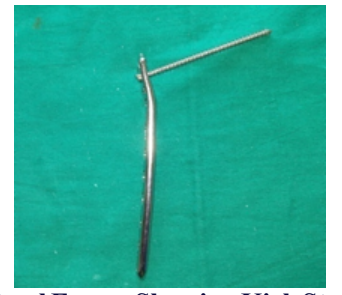

Case in which kick stand screw was used

Pre OPX-Ray

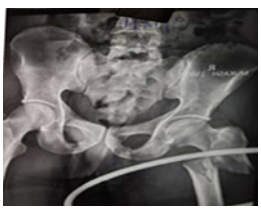

Immediate Post OPX-Ray

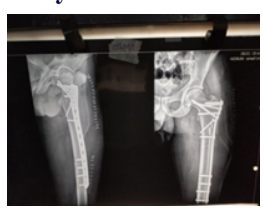

6 Month Follow up

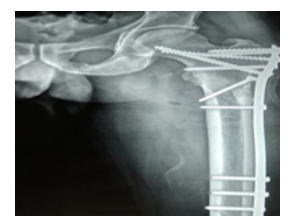

Case in which kick stand screw was not used Pre OPX-Ray

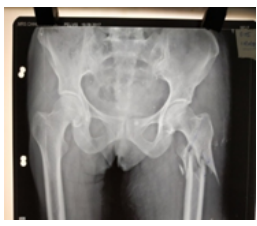

Immediate Post OPX-Ray

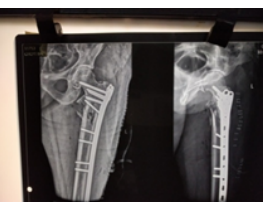

6 Month Follow up

APView Of Proximal Femur Showing Kick Stand Screw

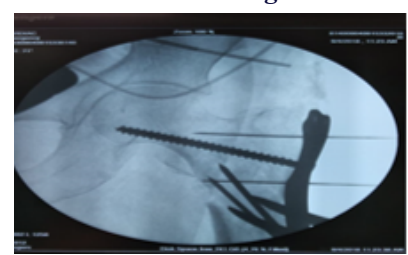

Postoperative details: Postoperatively all the patients kept in general ward, post op haematological investigation, postoperative $\mathrm{x}$ rays was obtained, dressing notes collected. From second post op day knee and hip physiotherapy was started and patients was allowed to sit on the bed. Average duration of inpatient care was 6 to 8 days. Patients strictly advised not to bear weight at least up to 3 months.

Follow up: Follow up was recorded at immediate post op, at discharge, at 1 month, at 3 month, at 6 month and at 1 year.

\section{Out Come Assessment:}

Clinical and functional outcome was assessed using Harris hip score pain was measured in terms of visual analogue score (vas). Radiological parameters assessed on $\mathrm{x}$-ray film on ap and lateral view including neck shaft angle, radiological signs of union (callus size, cortical bridging, progressive loss of fracture line). Clinical union was assessed by absence of pain at fracture site on weight bearing, absence of pain on palpation, ability to bear weight. Complications were assessed with patient's complaints, clinical examination, and with radiological examination.

Failure was defined by 10 degree change in neck shaft angle, any plate bending, any implant failure like screw breakdown, screw cut out ,screw back out, varus collapse, limb length discrepancy more than $2 \mathrm{~cm}$.

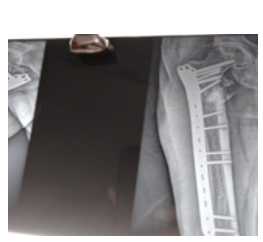

RESULT:

Union rate obtained was $80 \%$ in group without kick stand screw as compare to $94 \%$ in group with kick stand screw. Mean Harris hip score at the one year follow up was 71.2 in patient without kick stand screw as compare to 89.2 in patient with kick stand screw. Mean Visual analogue scale was 3.2 in first group as compare to 1.6 in second group at final follow up .Mean neck shaft angle at final follow-up was 120 degree in first group as compared to 135 degree in second group. Our study had shown that mean duration of surgery was 80.77 min, mean blood loss was $172.83 \mathrm{ml}$, mean fluoroscopy time 19.27 shoots per surgery, mean size of proximal incision was 7 and distal incision was $4.47 \mathrm{~cm}$, all these parameters are almost same in the both groups. Mean time of the clinical union was 15.2(14-18) in first group and 13.2 weeks (12-16 weeks) and mean time of the radiological union was $15.2(13-18)$ weeks and 12.80 weeks( $12-15$ weeks $)$. All patients allowed to bear weight at around 12 to 13 weeks.

Our study shown that kick stand screw placement result in better mechanical strength and fractures reduction was well maintained during follow up in patients with kick stand screw. These patients had better tolerability for weight bearing. So kick stand screw must be used in these fractures when fixed with DFLCP for better results.

\section{COMPLICATIONS:}

Group of patients without kick stand screw had three failures 2 in the form of screw break down and one in the form of plate bending, all three lend up in to varus deformity but the group of patient with kick stand screw had only one failure in the form of plate bending which 
was due to early weight bearing of the patient. First group had three patients with limb length shortening of $2 \mathrm{~cm}$ and in second group one patient had limb length shortening of $1 \mathrm{~cm}$.

Two Cases of complications in patients without kick stand screw. Pre OPX-Ray

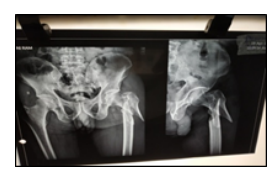

Immediate Post OPX-Ray

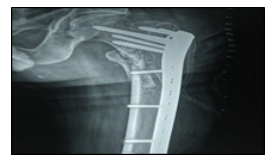

6 Month Follow up

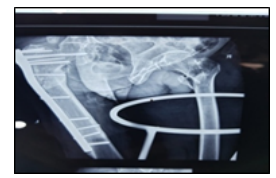

Pre OP X-Ray

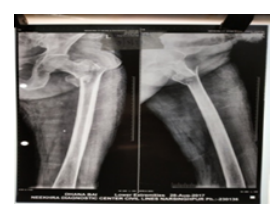

Immediate Post OP X-Ray

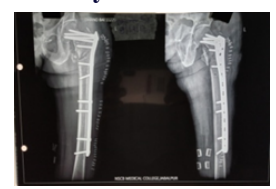

4 Month Follow up

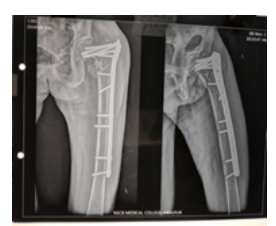

\section{DISCUSSION:}

The DFLCP has long been used for the distal femoral fractures. It is also a biomechanically sound implant for the subtrochanteric femoral fractures. Shape of the reversed contralateral DFLCP fits well with the contour of the proximal femur. Locking screws leave a gap between plate and bone which leaves periosteal blood supply intact. Problems with all the constructs of proximal femur plating are of mechanical strength, but when we put screw in the calcar region (kick stand screw) in the direction of neck it provides better mechanical strength to the construct and leads to better results.

Fracture reduction was well maintained during follow up in patients with kick stand screw. These patients had better tolerability for weight bearing. They had better Harris hip score at final follow up and lesser VAS score at final follow up. Kick stand screw had no role in duration of surgery, fluoroscopy time, blood loss, difficulty of reduction .

Philipp N. Streubel et $\mathbf{a l}^{14}$ analyzes Mechanical Failure After Locking Plate Fixation of Unstable Intertrochanteric Femur Fractures. They found that the presence of a "kickstand screw" and medial cortical reduction were not significantly different in cases with andwithout failure. A high rate of mechanical failure can be expected with proximal locking plate fixation of unstable proximal femur fractures. The use of a "kickstand" screw could not be established to reduce the risk for mechanical failure

Dr. Riyaz Babu Shaik et al $^{15}$ showed Proximal femoral locking plate: A good alternate in comminuted proximal femoral fractures.
They concluded that The PF-LCP is appropriate for complex and unstable proximal femoral fractures with poor bone quality. The PFLP with kickstand screw is biomechanically equivalent to the angled blade plate, but it allows for percutaneous insertion and avoids the potential morbidity of an extensile lateral approach of femur.

Rajesh Govindasamy et al $^{16}$ studied Proximal femur locking compression plate in complex proximal femoral fractures. Our study shows PF-LCP is a good alternative for treating complex proximal femoral fractures three proximal femoral locking screws including the "kickstand" screw should be inserted to increase the mechanical strength of the construct.

U Gunadham et al $^{17}$ studied the Outcome in Early Cases of Treatment of subtrochanteric Fractures with Proximal Femur Locking Compression Plate. They concluded that PF-LCP is an effective alternative treatment for subtrochanteric fractures when properly performed. The absence of kickstand screw was not associated with complications in their study.

R Gokul Nath et $\mathbf{a l}^{18}$ studied role of Proximal Femoral Locking Plate in treatment of subtrochanteric Fracture. This study showed that PFLP with the "kickstand" screw (that was applied at a $135^{\circ}$ in third hole of proximal part) was the stiffest construct $(92.2 \pm 17.4 \mathrm{Nm} / \mathrm{m})$. It was $194 \%$ stiffer than the PFLP without applying the kickstand screw, $211 \%$ stiffer than the angled blade plate and $309 \%$ stiffer than the broad locking plate. The precontoured structure of PF-LCP avoids varus collapse/ malreduction. The anatomical contouring of the implant to the lateral surface the proximal femur with its tip flush with tip of the greater trochanter restores neck shaft angle relationship, thus avoiding mal-union and mal-reduction.

Wei Ting Lee et $\mathbf{a l}^{19}$ analyzed Proximal femoral locking compression plate for proximal femoral fractures. The PF-LCP is appropriate for complex proximal femoral fractures with poor bone quality, revision surgeries, and multi-fragmentary subtrochanteric/proximal diaphyseal fractures. If the PF-LCP is used, the fracture must be adequately reduced and all proximal femoral locking screws (including the 'kickstand' screw) should be inserted to increase the mechanical strength of the construct.

Syed Ibrahim et $\mathbf{a l}^{20}$ showed retrospective analysis of surgicallytreated complex proximal femur fractures with proximal femoral locking compression plate

They showed that for better results with PF-LCP, the fracture must be adequately reduced and all proximal femoral locking screws (including the 'kick-stand' screw) should be inserted to increase the mechanical strength of the construct. PF-LCP locks the fracture in aposition without controlled collapse, so varus malalingment is avoided.

Brett D. et $\mathbf{a l}^{21}$ showed Biomechanical Comparison of Locked Plate Fixation With Percutaneous Insertion Capability Versus the Angled Blade Plate in a Subtrochanteric Fracture Gap Model Axial stiffness testing revealed that the PFLP with the "kickstand" screw was the stiffest construct $(92.2 \pm 17.4 \mathrm{Nm} / \mathrm{m})$, which was $211 \%$ stiffer than the blade plate, $309 \%$ stiffer than the broad plate, and $194 \%$ stiffer than the PFLP without the kickstand screw. The blade plate had the highest torsional stiffness $(2.42 \pm 0.08 \mathrm{Nm} /$ degree $)$, which was $151 \%$ stiffer than the broad plate, $128 \%$ stiffer than the PFLP with the kickstand, and $138 \%$ stiffer than the PFLP without the kickstand screw. The PFLP with the kickstand screw had the least irreversible deformation (6.3 $\mathrm{mm}$ ), which was $52 \%$ less than the study revealed that the PFLP with the "kickstand" provides more axial stiffness, less torsional stiffness, and equivalent irreversible deformation to cyclic axial loading when compared with the blade plate.

Our study also showed results better in patients with kick stand screw.

In our study cases in which kick stand screw was used they showed better mechanical strength and gave better clinical results. So in proximal femur fractures including sub trochanteric fracture fixation 'kick stand screw' must be used whether DFLCP or PFLCP is used. One problem with the plating is that early weight bearing must be avoided to prevent plate failure but weight bearing is better tolerated by plating with kick stand screw. 
REFERENCE

1. Marsh JL, Saltzman CL, Bucholz R, Heckman J. Rockwood and Green's fractures in adults. 2001;2(6):827-1844

2. McLaurin TM, Lawler EA. Treatment modalities for subtrochanteric fractures in the elderly. Techniques in Orthopaedics. 2004;19(3):197-213

3. Baumgaertner MR. The pertrochanteric external fixator reduced pain, hospital stay, and mechanical complications in comparison with the sliding hip screw. J Bone Joint Surg Am 2002; 84: 1488

4. Adams CI, Robinson CM, Court-Brown CM, Mc Queen MM. Prospective randomized controlled trial of an intramedullary nail versus dynamic screw and plate for intertrochanteric fractures of the femur. J Orthop Trauma 2001; 15: 394-400

5. Winquist RA, Hansen ST, Clawson DK:Closed intramedullary nailing of femoral fractures - a report of five hundred and twenty cases. J Bone Joint Surg Am 2001; 83fractures - a report of five hundred and twenty cases. J Bone Joint Surg Am 2001; 83A:1912

6. Kakkar R, Kumar S, Singh A: Cephalomedullary nailing for proximal femoral fractures. Int Orthop 2005; 29:21-24

7. J Schatzker, Mahomed N, Schiffman K, et al. Dynamic condylar screw: a new device. A preliminar. J Orthop Trauma 1989;3:124-32.

8. Wolfgang GL, BryantMH, O'Neill JP. Treatment of intertrochanteric fracture of the femur using sliding screw plate fixation. Clin Orthop 1982;163: 148-58.

9. Yoo MC, Cho YJ, Kim KI, et al. Treatment of unstable peritrochanteric femoral fractures using a 95 degrees angled blade plate. J Orthop Trauma 2005;19:687-92

10. van Meeteren MC, van Rief YE, Roukema JA. Condylar plate fixation of subtrochanteric femoral fractures. Injury. 1996;27:715e71.

11. Yao C, Zhang CQ, Jin DX, Chen YF (2011) Early results of reverse less invasive stabilization system plating in treating elderly intertrochanteric fractures: a prospective study compared to proximal femoral nail. Chin Med J (Engl) 124:2150-215

12. Zhou F, Zhang ZS, Yang H et al (2012) Less invasive stabilization system (LISS) versus proximal femoral nail anti-rotation (PFNA) in treating proximal femoral fractures: a prospective randomized study. J Orthop Trauma 26:155-162.

13. Tao R, Lu Y, Xu H, Zhou ZY, Wang YH, Liu F (2013). Internal fixation of intertrochanteric hip fractures: a clinical comparison of two implant designs. Scientific World J: 834825

14. Philipp N. Streubel, MD, Michael J. Moustoukas, MD, and William T. Obremskey, MD, MPH(2013). Mechanical Failure After Locking Plate Fixation of Unstable Intertrochanteric Femur Fractures.J Orthop Trauma 2013;27:22-28

15. Dr. Riyaz Babu Shaik, Dr. Satya Kumar K and Dr. Cherukuri Akhila Sai Sree Proximal femoral locking plate: A good alternate in comminuted proximal femoral fractures. International Journal of Orthopaedics Sciences 2017;3(4): 937-940

16. Rajesh Govindasamy, Ramkumar Gnanasundaram, Saravanan Kasirajan, Jimmy J. Meleppuram, Kumar Archit. Proximal femur locking compression plate in complex proximal femoral fractures: a retrospective analysis. Int J Res Orthop. 2016 Sep;2(3):104-108

17. U Gunadham*, MD, J Jampa, MD, S Suntornsup, MD, B Leewiriyaphun, MD The Outcome in Early Cases of Treatment of Subtrochanteric Fractures with Proximal Femur Locking Compression Plate Malaysia Orthopaedic Journal 2014 Vol 8 No 2

18. R Gokul Nath, Sah Ansari. Role of Proximal Femoral Locking Plate in treatment of Subtrochanteric Fractures; Case series. MedPulse International Journal of Orthopedics. July 2017;3(1): 01-07.

19. Wei Ting Lee, Diarmuid Murphy, Fareed HY Kagda, Joseph Thambiah. Proximal femoral locking compression plate for proximal femoral fractures. Journal of Orthopaedic Surgery 2014;22(3):287-93

20. Syed Ibrahim , Jimmy Joseph Meleppuram. A retrospective analysis of surgicallytreated complex proximal femur fractures with proximal femoral locking compression plate. Revbrasortop. $2017 ; 52(6): 644-650$

21. Brett D. Crist, MD, Afshin Khalafi, MD, Scott J. Hazelwood, PhD, and Mark A. Lee, Brett D. Crist, MD, Afshin Khalafi, MD, Scott J. Hazelwood, PhD, and Mark A. Lee,
MD. A Biomechanical Comparison of Locked Plate Fixation With Percutaneous MD. A Biomechanical Comparison of Locked Plate Fixation With Percutaneous
Insertion Capability Versus the Angled Blade Plate in a Subtrochanteric Fracture Gap Model 